\title{
PERIODIC SOLUTIONS OF DISSIPATIVE SYSTEMS REVISITED
}

\author{
JAN ANDRES AND LECH GÓRNIEWICZ
}

Received 23 June 2005; Revised 4 October 2005; Accepted 17 October 2005

We reprove in an extremely simple way the classical theorem that time periodic dissipative systems imply the existence of harmonic periodic solutions, in the case of uniqueness. We will also show that, in the lack of uniqueness, the existence of harmonics is implied by uniform dissipativity. The localization of starting points and multiplicity of periodic solutions will be established, under suitable additional assumptions, as well. The arguments are based on the application of various asymptotic fixed point theorems of the Lefschetz and Nielsen type.

Copyright (c) 2006 J. Andres and L. Górniewicz. This is an open access article distributed under the Creative Commons Attribution License, which permits unrestricted use, distribution, and reproduction in any medium, provided the original work is properly cited.

\section{Introduction}

Consider the system

$$
x^{\prime}=F(t, x), \quad F(t, x) \equiv F(t+\tau, x), \tau>0,
$$

where $F:[0, \tau] \times \mathbb{R}^{n} \rightarrow \mathbb{R}^{n}$ is a Carathéodory function.

We say that system (1.1) is dissipative (in the sense of Levinson [23]) if there exists a common constant $D>0$ such that

$$
\limsup _{t \rightarrow \infty}|x(t)|<D
$$

holds, for all solutions $x(\cdot)$ of $(1.1)$.

Theorem 1.1 (classical). Assume the uniqueness of solutions of (1.1). If system (1.1) is dissipative, then it admits a $\tau$-periodic solution $x(\cdot) \in A C\left([0, \tau], \mathbb{R}^{n}\right)($ with $|x(t)|<D$, for all $t \in \mathbb{R})$.

The standard proof of Theorem 1.1 (see, e.g., [30, pages 172-173]) is based on the application of Browder's fixed point theorem [7], jointly with the fact that, in the case of 
uniqueness, time periodic dissipative systems are uniformly dissipative, that is,

$$
\forall D_{1}>0 \quad \exists \Delta t>0:\left[t_{0} \in \mathbb{R},\left|x_{0}\right|<D_{1}, t \geq t_{0}+\Delta t\right] \Longrightarrow|x(t)|<D_{2},
$$

where $D_{2}>0$ is a common constant, for all $D_{1}>0$, and $x(\cdot)=x\left(\cdot, t_{0}, x_{0}\right)$ is a solution of $(1.1)$ such that $x\left(t_{0}\right)=x\left(t_{0}, t_{0}, x_{0}\right)=x_{0} \in \mathbb{R}^{n}$, and that their solutions are uniformly bounded (see [26]).

Let us note that the same idea of the proof was already present in [9], but since that time Browder's theorem was not at our disposal, only subharmonic (i.e., $k \tau$-periodic; $k \in$ $\mathbb{N}$ ) solutions were deduced by means of the Brouwer fixed point theorem (cf. also [27]). So far, many extensions of Theorem 1.1 were obtained especially for abstract dissipative processes or in infinite dimensions (see, e.g., $[1,2,4,6,8,10,14,19-22,30]$ ).

The aim of this paper is first to reprove Theorem 1.1 in an extremely simple way by means of asymptotic fixed point theorems and to demonstrate that a very recent theorem of this type in [28] is only a very particular case of much older results, for example, in $[11-13,24,25]$ (cf. also [2, 18]). Furthermore, we will obtain more precise information about localization of the starting point of the implied $\tau$-periodic solution of (1.1) by means of the asymptotic relative Lefschetz theorem [17], and discuss possible multiplicity results by means of the asymptotic relative Nielsen theorem [5]. Finally, we will generalize Theorem 1.1, jointly with the relative and multiplicity results, in the lack of uniqueness.

\section{Asymptotic fixed point theorems}

All proofs of Theorem 1.1 are via the Poincaré translation operator $T_{\tau}: \mathbb{R}^{n} \rightarrow \mathbb{R}^{n}$ along the trajectories of (1.1), defined as follows:

$$
T_{\tau}\left(x_{0}\right):=\left\{x(\tau) \mid x(\cdot) \text { is a solution of (1.1) with } x(0)=x_{0}\right\} ; \quad x_{0} \in \mathbb{R}^{n} .
$$

Since uniqueness implies the continuous dependence of solutions of (1.1) on initial values (cf., e.g., [2]), $T_{\tau}$ is completely continuous such that

$$
T_{\tau}^{k}\left(x_{0}\right) \equiv T_{k \tau}\left(x_{0}\right)
$$

Moreover, dissipativity (cf. condition (1.2)) implies that

$$
\limsup _{k \rightarrow \infty}\left|T_{\tau}^{k}\left(x_{0}\right)\right|<D \quad \forall x_{0} \in \mathbb{R}^{n}
$$

by which

$$
\left\{x_{0}, T_{\tau}\left(x_{0}\right), \ldots, T_{\tau}^{m}\left(x_{0}\right), \ldots\right\} \cap W \neq \varnothing \quad \forall x_{0} \in \mathbb{R}^{n}
$$

where $W:=\left\{x_{0} \in \mathbb{R}^{n}|| x_{0} \mid \leq D\right\}$ is a compact window (cf. below).

Because of an apparent one-to-one correspondence between $\tau$-periodic solutions $x(\cdot)$ of (1.1) and fixed points $x_{0}$ of $T_{\tau}$, we need an (asymptotic) fixed point theorem such that a continuous self-map of $\mathbb{R}^{n}$ with a compact window would guarantee a fixed point. This formulation exactly corresponds to the fixed point theorem in [28]. 
Hence, let us start with this theorem and its generalizations in a more precise way.

We will assume that all considered topological spaces are metric and all mappings between such spaces are continuous.

Let $f: X \rightarrow X$ be a continuous map and let $x \in X$. Then the set

$$
O(x)=\left\{x, f(x), \ldots, f^{m}(x), \ldots\right\}
$$

is called the orbit of $x$ under $f$.

A (compact) set $W \subset X$ is called a window for $f$ if, for every $x \in X$, we have

$$
O(x) \cap W \neq \varnothing \text {. }
$$

In [28], the following main theorem was proved.

Theorem 2.1. If $f: \mathbb{R}^{n} \rightarrow \mathbb{R}^{n}$ is a continuous map which possesses a compact window, then

$$
\operatorname{Fix}(f)=\left\{x \in \mathbb{R}^{n} \mid f(x)=x\right\} \neq \varnothing .
$$

Hence, Theorem 1.1 is a direct consequence of Theorem 2.1 applied to $T_{\tau}$ defined in (2.1). On the other hand, Theorem 2.1 is only a very special case of several asymptotic fixed point theorems published a long time before [28]. We will briefly recall some of these theorems with comments.

2.1. Mappings with compact attractors. Following Nussbaum ([24, 25]; see also $[2,11-$ $13,15,16,18]$ ), we say that a (compact) set $A \subset X$ is an attractor for $f: X \rightarrow X$ if, for every $x \in X$, we have

$$
\overline{O(x)} \cap A \neq \varnothing,
$$

where $\overline{O(x)}$ denotes the closure of $O(x)$ in $X$.

Remark 2.2. Every window for $f: X \rightarrow X$ is apparently an attractor for $f$. Moreover, let us observe that, for example, any contraction $f: \mathbb{R}^{n} \rightarrow \mathbb{R}^{n}$ (or, more generally, the contraction $f: X \rightarrow X$, where $X$ is a complete metric space) admits an attractor, but not necessarily a window.

We recall that a map $f: X \rightarrow X$ is locally compact if, for every $x \in X$, there exists an open neighbourhood $U_{x}$ of $x$ in $X$ such that $\overline{f\left(U_{x}\right)}$ is compact.

Remark 2.3. Obviously, if $X$ is a locally compact space (in particular, if $X=\mathbb{R}^{n}$ ), then any continuous map $f: X \rightarrow X$ is locally compact.

Let us still recall two notions introduced by Borsuk (see $[2,15]$ or [18]).

A space $X$ is called absolute neighbourhood retract (ANR, for short) if there exists an open set $U$ of a normed space $E$ which $r$-dominates $X$, that is, if there are continuous mappings $r: U \rightarrow X$ and $s: X \rightarrow U$ such that $r \circ s=\mathrm{id}_{X}$. If, in particular, a space $X$ is homeomorphic to a neighbourhood retract in $\mathbb{R}^{n}$, then we speak about a Euclidean neighbourhood retract (ENR). Obviously, ENR $\subset$ ANR. If $U=E$ is a normed space which $r$-dominates $X$, then $X$ is called an absolute retract (AR). 
Remark 2.4. Evidently, AR $\subset \mathrm{ANR}$, and every normed space is an absolute retract.

In 1975 Fournier [11-13] proved the following.

Theorem 2.5. If $X$ is an ANR-space and $f: X \rightarrow X$ is a locally compact map with compact attractor, then

(i) the (generalized) Lefschetz number $\Lambda(f)$ of $f$ is well defined, and

(ii) $\Lambda(f) \neq 0$ implies that $\operatorname{Fix}(f) \neq \varnothing$.

As an immediate consequence of Theorem 2.5, we obtain the following.

Corollary 2.6. If $X$ is a locally compact ANR and $f: X \rightarrow X$ is a map with compact attractor, then

(i) the generalized Lefschetz number $\Lambda(f)$ of $f$ is well-defined;

(ii) $\Lambda(f) \neq 0$ implies that $\operatorname{Fix}(f) \neq \varnothing$.

Since every AR-space is contractible, we infer that $\Lambda(f)=1$, for an arbitrary $f: X \rightarrow X$, and so from Theorem 2.5 (or Corollary 2.6), we obtain the following corollary.

Corollary 2.7. If $X \in \mathrm{AR}$ ( $X$ is a locally compact $A R$-space), then every locally compact map with compact attractor (every map with compact attractor) $f: X \rightarrow X$ has a fixed point.

Remark 2.8. Observe that Corollary 2.7 is a far generalization of Theorem 2.1 in the introduction. Let us also note that the idea of Corollary 2.7 is, in fact, already present in the mentioned Theorem 2.1 and in [7] published in 1959.

2.2. Compact absorbing contractions. Theorem 2.5 is not the most general known result. We recall (see $[2,15,18]$ ) that a continuous map $f: X \rightarrow X$ is called a compact absorbing contraction (written, $f \in \mathrm{CAC}(X))$ if there exists an open subset $U \subset X$ such that the following conditions are satisfied:

(i) $O(x) \cap U \neq \varnothing$, for every $x \in X$,

(ii) $f(U) \subset U$, We let

(iii) the map $\tilde{f}: U \rightarrow U, \tilde{f}(x):=\left.f(x)\right|_{x \in U}$, is compact.

$$
\begin{aligned}
& \mathrm{CA}(X)=\{f: X \rightarrow X \mid f \text { is continuous with compact attractor }\}, \\
& \mathrm{CA}_{0}(X)=\{f: X \rightarrow X \mid f \text { is continuous and locally compact with compact attractor }\} .
\end{aligned}
$$

It is well known (see $[2,16,18])$ that

$$
\mathrm{CA}_{0}(X) \subset \mathrm{CAC}(X) \subset \mathrm{CA}(X)
$$

and that both of the above inclusions are proper.

Remark 2.9. We would like to point out that Theorem 2.5 and Corollaries 2.6, 2.7 can be reformulated for CAC-mappings (see again $[2,16,18]$ ). 
Let us recall the following old open problem.

Open problem 2.10. Is it possible to prove Theorem 2.5 (or Corollaries 2.6, 2.7) for CAmappings?

2.3. Condensing mappings. Some further results being a far generalization of Theorem 2.1 will still be mentioned here.

Let $E$ be a Banach space and let

$$
B(E)=\{A \subset E \mid A \text { is a bounded subset of } E\} .
$$

By $\alpha: B(E) \rightarrow[0, \infty)$, we denote a measure of noncompactness (see $[2,15,16]$ or [25]). For the sake of simplicity, we can assume that $\alpha$ is the Kuratowski measure of noncompactness. Let $X \subset E$ and $f: X \rightarrow X$ be a continuous map. We say that $f$ is a condensing map if, for every bounded $A \subset X$ with $\alpha(A)>0$, we have

$$
\alpha(f(A))<\alpha(A)
$$

Nussbaum $[24,25]$ proved the following theorem.

Theorem 2.11. Let $X$ be an open subset of $E$ and let $f: X \rightarrow X$ be a condensing map with compact attractor. Then

(i) the (generalized) Lefschetz number $\Lambda(f)$ of $f$ is well defined,

(ii) $\Lambda(f) \neq 0$ implies that $\operatorname{Fix}(f) \neq \varnothing$.

We say that a closed bounded subset $X$ of $E$ is a special ANR (see [16] or [2]) if there exist an open $U \subset E$ and a continuous map $r: U \rightarrow X$ such that:

(i) $X \subset U$,

(ii) $r(x)=x$, for every $x \in X$,

(iii) for every $A \subset U$, we have $\alpha(r(A)) \leq \alpha(A)$.

In [16], the following result was proved.

Theorem 2.12. Let $X$ be a special ANR and let $f: X \rightarrow X$ be a condensing map. Then

(i) the (generalized) Lefschetz number $\Lambda(f)$ of $f$ is well defined,

(ii) $\Lambda(f) \neq 0$ implies that $\operatorname{Fix}(f) \neq \varnothing$.

Remark 2.13. Since, according to [29], the Nielsen number $N(f)$ for a single valued continuous map $f: X \rightarrow X$ is well defined, provided

(i) $X$ is an ANR,

(ii) $\operatorname{Fix}(f)$ is compact,

(iii) $\Lambda(f)$ is well defined,

the above conclusions can be completed by the cardinality \# $\operatorname{Fix}(f) \geq N(f)$.

\section{Some further information}

Although all theorems from the foregoing section generalize Theorem 2.1, none of them would bring new information when they are applied to prove Theorem 1.1. Thus, in order to obtain some further information like a more precise localization of the starting point 
of the implied $\tau$-periodic solution of (1.1) or a lower estimate of the number of $\tau$-periodic solutions of (1.1), we need more advanced relative fixed point theorems.

The following version of relative Lefschetz theorem is due to the second author and Granas [17] (cf. [2, 15]).

Theorem 3.1. Let $X$ and $X_{0} \subset X$ be ANR-spaces and let $f:\left(X, X_{0}\right) \rightarrow\left(X, X_{0}\right)$ be a CACmap, that is, let $\left.f\right|_{X}: X \rightarrow X$ and $\left.f\right|_{X_{0}}: X_{0} \rightarrow X_{0}$ be CAC-maps. Then the relative Lefschetz number $\Lambda(f)$ for $f$ is well defined and satisfies the equality

$$
\Lambda(f)=\Lambda\left(\left.f\right|_{X}\right)-\Lambda\left(\left.f\right|_{X_{0}}\right)
$$

where $\Lambda\left(\left.f\right|_{X}\right)$ and $\Lambda\left(\left.f\right|_{X_{0}}\right)$ are the (well defined; see above) generalized Lefschetz numbers of $\left.f\right|_{X}$ and $\left.f\right|_{X_{0}}$, respectively. Moreover, if $\Lambda(f) \neq 0$, that is, if $\Lambda\left(\left.f\right|_{X}\right) \neq \Lambda\left(\left.f\right|_{X_{0}}\right)$, then there exists a fixed point $x \in \operatorname{Fix}(f)$ such that $x \in \overline{X \backslash X_{0}}$.

In view of (2.10), we can get immediately the following.

Corollary 3.2. Let $X$ and $X_{0} \subset X$ be ANR-spaces and let $f \in \mathrm{CA}_{0}\left(\left(X, X_{0}\right)\right)$, that is, let $\left.f\right|_{X}: X \rightarrow X$ and $\left.f\right|_{X_{0}}: X_{0} \rightarrow X_{0}$ be locally compact maps with compact attractors. If

$$
\Lambda\left(\left.f\right|_{X}\right) \neq \Lambda\left(\left.f\right|_{X_{0}}\right)
$$

then there exists a fixed point $x \in \operatorname{Fix}(f)$ such that $x \in \overline{X \backslash X_{0}}$.

Now, assume that (1.1) is dissipative (i.e., (1.2) holds, for all solutions $x(\cdot)$ of (1.1)) and that a compact ENR-set $A \subset \mathbb{R}^{n}$ exists such that $x(0) \in A$ implies $x(t) \in A$, for all $t \in[0, \tau]$. Since $\left.T_{\tau}\right|_{\mathbb{R}^{n}} \in \mathrm{CA}_{0}\left(\mathbb{R}^{n}\right),\left.T_{\tau}\right|_{A}$ is a compact map and $\mathbb{R}^{n} \in \mathrm{AR}$, the generalized Lefschetz numbers $\Lambda\left(\left.T_{\tau}\right|_{\mathbb{R}^{n}}\right), \Lambda\left(\left.T_{\tau}\right|_{A}\right)$ are well defined satisfying

$$
\Lambda\left(\left.T_{\tau}\right|_{\mathbb{R}^{n}}\right)=1, \quad \Lambda\left(\left.T_{\tau}\right|_{A}\right)=\Lambda\left(\left.\mathrm{id}\right|_{A}\right)=\chi(A),
$$

where $\chi(A)$ denotes the Euler characteristic of $A$. Hence, Theorem 1.1 can be improved by means of Corollary 3.2 as follows.

Corollary 3.3. Assume the uniqueness of solutions $x(\cdot)$ of (1.1). Assume also that there exists a compact ENR-set $A \subset \mathbb{R}^{n}$ with $\chi(A) \neq 1$ such that $x(0) \in A$ implies $x(t) \in A$, for all $t \in[0, \tau]$. If system (1.1) is dissipative, then it admits a $\tau$-periodic solution $x_{0}(\cdot)$ with $x_{0}(t) \in \mathscr{D}$, for all $t \in \mathbb{R}$, and with $x_{0}(0) \in \mathscr{D} \backslash \operatorname{int} A$, where $\mathscr{D}:=\left\{x_{0} \in \mathbb{R}^{n}|| x_{0} \mid<D\right\}$.

With respect to the multiplicity, we have at our disposal the following very recent theorem due to the first author and Wong [5].

Theorem 3.4. Let $X$ and $X_{0} \subset X$ be ANR-spaces and let $f:\left(X, X_{0}\right) \rightarrow\left(X, X_{0}\right)$ be a CACmap, that is, let $\left.f\right|_{X}: X \rightarrow X$ and $\left.f\right|_{X_{0}}: X_{0} \rightarrow X_{0}$ be CAC-maps. Then the relative Nielsen number $N\left(f ; X, X_{0}\right)$ for $f$ (on the total space) is well defined and satisfies the equality

$$
N\left(f ; X, X_{0}\right)=N\left(\left.f\right|_{X}\right)+N\left(\left.f\right|_{X_{0}}\right)-N\left(\left.f\right|_{X},\left.f\right|_{X_{0}} ; X, X_{0}\right),
$$

where $N\left(\left.f\right|_{X}\right)$ and $N\left(\left.f\right|_{X_{0}}\right)$ are the (well defined; see Remark 2.9) Nielsen numbers of $\left.f\right|_{X}$ and $\left.f\right|_{X_{0}}$, respectively, while $N\left(\left.f\right|_{X},\left.f\right|_{X_{0}} ; X, X_{0}\right)$ denotes the number of essential common 
Nielsen classes of $\left.f\right|_{X}$ and $\left.f\right|_{X_{0}}$ (for the definitions and more details, see [5]). Moreover,

$$
0 \leq N\left(\left.f\right|_{X}\right) \leq N\left(f ; X, X_{0}\right) \leq \# \operatorname{Fix}\left(\left.f\right|_{X}\right)
$$

that is, $N\left(f ; X, X_{0}\right)$ provides a lower estimate of the number of fixed points of $f$ on the total space $X$ and it is a CAC-homotopy invariant (jointly in $X \times X_{0} \times[0,1]$ ).

In view of (2.10), we can get immediately the following.

Corollary 3.5. Let $X$ and $X_{0} \subset X$ be ANR-spaces and let $f \in \mathrm{CA}_{0}\left(\left(X, X_{0}\right)\right)$, that is, let $\left.f\right|_{X}: X \rightarrow X$ and $\left.f\right|_{X_{0}}: X_{0} \rightarrow X_{0}$ be locally compact maps with compact attractors. Then every map $g:\left(X, X_{0}\right) \rightarrow\left(X, X_{0}\right)$ which is $\mathrm{CA}_{0}$-homotopic (jointly in $\left.X \times X_{0} \times[0,1]\right)$ with $f$ $(f \sim g)$ admits at least $\left[N\left(\left.f\right|_{X}\right)+N\left(\left.f\right|_{X_{0}}\right)-N\left(\left.f\right|_{X},\left.f\right|_{X_{0}} ; X, X_{0}\right)\right]$ fixed points on the total space $X$.

Now, assume again that (1.1) is dissipative (i.e., (1.2) holds, for all solutions $x(\cdot)$ of (1.1)) and that a compact ENR-set $A \subset \mathbb{R}^{n}$ exists such that $x(0) \in A$ implies $x(t) \in A$, for all $t \in[0, \tau]$. Since $\left.T_{\tau}\right|_{\mathbb{R}^{n}} \in \mathrm{CA}_{0}\left(\mathbb{R}^{n}\right),\left.T_{\tau}\right|_{A}$ is a compact map and $\mathbb{R}^{n} \in \mathrm{AR}$, the relative Nielsen number $N\left(T_{\tau} ; \mathbb{R}^{n}, A\right)$ is well defined satisfying

$$
0 \leq N\left(T_{\tau} ; \mathbb{R}^{n}, A\right)=N\left(\left.T_{\tau}\right|_{\mathbb{R}^{n}}\right)+N\left(\left.T_{\tau}\right|_{A}\right)-N\left(\left.T_{\tau}\right|_{\mathbb{R}^{n}},\left.T_{\tau}\right|_{A} ; \mathbb{R}^{n}, A\right),
$$

where $N\left(\left.T_{\tau}\right|_{\mathbb{R}^{n}}\right)=1$ and $N\left(\left.T_{\tau}\right|_{A}\right)=N\left(\left.\mathrm{id}\right|_{A}\right)$. Thus,

$$
N\left(\left.T_{\tau}\right|_{\mathbb{R}^{n}},\left.T_{\tau}\right|_{A} ; \mathbb{R}^{n}, A\right) \in\{0,1\},
$$

and subsequently

$$
N\left(T_{\tau} ; \mathbb{R}^{n}, A\right)= \begin{cases}1 & \text { if } N\left(\left.T_{\tau}\right|_{\mathbb{R}^{n}},\left.T_{\tau}\right|_{A} ; \mathbb{R}^{n}, A\right)=1, \\ 1+N\left(\left.\mathrm{id}\right|_{A}\right) & \text { if } N\left(\left.T_{\tau}\right|_{\mathbb{R}^{n}},\left.T_{\tau}\right|_{A} ; \mathbb{R}^{n}, A\right)=0 .\end{cases}
$$

In view of (3.8), Corollary 3.5 can be applied via $T_{\tau}:\left(\mathbb{R}^{n}, A\right) \rightarrow\left(\mathbb{R}^{n}, A\right)$ as follows.

Corollary 3.6. Assume the uniqueness of solutions $x(\cdot)$ of (1.1). Assume also that there exists a compact ENR-set $A \subset \mathbb{R}^{n}$ such that $x(0) \in A$ implies $x(t) \in A$, for all $t \in[0, \tau]$. If system (1.1) is dissipative (i.e., (1.2) holds), then it admits at least $1+N\left(\left.\mathrm{id}\right|_{A}\right) \tau$-periodic solutions, provided there is no common essential Nielsen class of $\left.T_{\tau}\right|_{\mathbb{R}^{n}}$ and $\left.T_{\tau}\right|_{A}$.

Remark 3.7. The nonrelative Nielsen number (cf. Remark 2.9) is equal to 1, and so, would not help here. Similarly, the relative Nielsen numbers on the complement and on the closure of the complement defined in [5] are trivially equal to 0 or 1.

\section{Lack of uniqueness}

In the lack of uniqueness, one usually applies the standard limiting argument, provided $F:[0, \tau] \times \mathbb{R}^{n} \rightarrow \mathbb{R}^{n}$ is continuous. $F$ can be namely approximated with an arbitrary accuracy by a locally Lipschitz map which leads again to the uniqueness of solutions 
of approximating differential systems. If these systems are assumed to be dissipative, then they admit, according to Theorem 1.1, $\tau$-periodic solutions. The desired $\tau$-periodic solution of (1.1) can be so obtained, by the diagonalization argument, as a uniform limit of a selected sequence of $\tau$-periodic solutions of approximating systems. In case of Carathéodory right-hand sides, one can regularize $F(\cdot, x)$ by an arbitrarily "close" continuous $\widetilde{F}(\cdot, x)$ at first, and then apply the standard limiting argument to a selected sequence of $\tau$-periodic solutions of approximating regularized systems, provided they are dissipative.

On the other hand, we can proceed more directly. First of all, we know that the (multivalued) Poincaré translation operator $T_{\tau}: \mathbb{R}^{n} \multimap \mathbb{R}^{n}$ (i.e., $T_{\tau}: \mathbb{R}^{n} \rightarrow 2^{\mathbb{R}^{n}} \backslash\{\varnothing\}$ ) is admissible in the sense of the second author. More precisely, it is an upper semicontinuous composition of an $R_{\delta}$-mapping with a single-valued continuous mapping (for the definitions and more details, see $[2,15])$. Furthermore, if (1.1) is uniformly dissipative (i.e., (1.3) holds, for all solutions $x(\cdot)$ of $(1.1)$ ), then for every $x_{0} \in \mathbb{R}^{n}$, there certainly exists $m=m_{x_{0}}$ such that $T_{\tau}^{k}\left(x_{0}\right) \subset U$, for every $k \geq m$, where $U$ is an (arbitrary) open neighbourhood of a compact attractor $\left\{x_{0} \in \mathbb{R}^{n}|| x_{0} \mid \leq D_{2}\right\}$, which we write as $T_{\tau} \in \mathbb{C A}_{0}\left(\mathbb{R}^{n}\right)$. Thus, since an analogy of condition (2.10) holds for multivalued admissible maps, the following version of an asymptotic Lefschetz theorem can be applied to $T_{\tau}$ for obtaining a $\tau$-periodic solution of (1.1) (see [2, pages 98-99]).

Theorem 4.1. If $X \in \mathrm{ANR}$ and $\varphi \in \mathbb{C A}_{0}(X)$, that is, $\varphi: X \multimap X$ is a locally compact admissible mapping with a compact attractor, in the above sense, then

(i) the Lefschetz set $\Lambda(\varphi)$ is well defined,

(ii) $\Lambda(\varphi) \neq\{0\}$ implies that $\operatorname{Fix}(\varphi):=\left\{x \in \mathbb{R}^{n} \mid x \in \varphi(x)\right\} \neq \varnothing$.

If, in particular, $X \in \mathrm{AR}$, then $\Lambda(\varphi)=\{1\}$, and so $\varphi$ admits a fixed point.

Since $\mathbb{R}^{n} \in \mathrm{AR}$ and $T_{\tau} \in \mathbb{C A}_{0}\left(\mathbb{R}^{n}\right)$, we obtain as an immediate consequence of Theorem 4.1 that $\operatorname{Fix}\left(T_{\tau}\right) \neq \varnothing$, and subsequently that uniformly dissipative system (1.1) admits a $\tau$-periodic solution.

Since we also have to our disposal (multivalued) $\mathbb{C A}_{0}$-versions of Corollaries 3.2 and 3.5 (see [3] and cf. also [2, Chapter II.5]), with the additional restriction imposed on $A \subset$ $\mathbb{R}^{n}$ in the Nielsen case, namely that $A$ is still assumed there to be closed and connected, we can summarize our discussion as follows.

Theorem 4.2. Uniformly dissipative system (1.1) admits a $\tau$-periodic solution. Furthermore, if a compact ENR-set $A \subset \mathbb{R}^{n}$ exists such that $x(0) \in A$ implies $x(t) \in A, t \in[0, \tau]$, for solutions $x(\cdot)$ of (1.1), then uniformly dissipative system (1.1) admits a $\tau$-periodic solution $x_{0}(\cdot)$ with $x_{0}(0) \in \mathscr{D} \backslash \operatorname{int} A$, where $\mathscr{D}:=\left\{x_{0} \in \mathbb{R}^{n}|| x_{0} \mid<D_{2}\right\}$ and $D_{2}>0$ is a constant in (1.3), provided $\chi(A) \neq 1$. If $A$ is still connected (in the case of uniqueness, it is not necessary), then uniformly dissipative system (1.1) admits at least $1+N\left(\left.\mathrm{id}\right|_{A}\right) \tau$-periodic solutions, provided there is no common essential Nielsen class of $\left.T_{\tau}\right|_{\mathbb{R}^{n}}$ and $\left.T_{\tau}\right|_{A}$.

Example 1. Taking in Theorem $4.2 A \subset \mathbb{R}^{n}$ such that $A=A_{1} \cup A_{2}$ and $A_{1} \cap A_{2}=\varnothing$, where both $A_{1}, A_{2}$ are compact subinvariant absolute retracts, we have $\chi(A)=\chi\left(A_{1}\right)+$ $\chi\left(A_{2}\right)=2$, and so the dissipative system (1.1) admits a $\tau$-periodic solution $x_{0}(\cdot)$ with $x_{0}(0) \in \mathscr{D} \backslash \operatorname{int} A$. In the case of uniqueness, the dissipative system (1.1) admits at least 
three $\tau$-periodic solutions, because $1+N\left(\left.\mathrm{id}\right|_{A}\right)=1+N\left(\left.\mathrm{id}\right|_{A_{1}}\right)+N\left(\left.\mathrm{id}\right|_{A_{2}}\right)=3$, and there is evidently no common essential Nielsen class of $\left.T_{\tau}\right|_{\mathbb{R}^{n}}$ and $\left.T_{\tau}\right|_{A}$.

Remark 4.3. Since, in the case of uniqueness, dissipativity (cf. (1.2)) implies uniform dissipativity (cf. (1.3)) of (1.1), we can assume without any loss of generality uniform dissipativity, instead of dissipativity, of (1.1). Therefore, Theorem 4.2 is indeed a generalization of Theorem 1.1 and Corollaries 3.3, 3.6, provided $A \subset \mathbb{R}^{n}$ in Corollary 3.6 is still connected. On the other hand, for a connected $A$ in Theorem $4.2, N\left(\left.\mathrm{id}\right|_{A}\right)=0$ holds only.

\section{Concluding remarks}

Uniform dissipativity of (1.1) and positive flow-invariance of $A$ can be expressed in terms of respective guiding and bounding (Liapunov) functions in the following way (for more details, see $[2,30])$.

Proposition 5.1. Let a locally Lipschitz (guiding) function $V: \mathbb{R}^{n} \rightarrow \mathbb{R}$ exist such that

(i) $\lim _{|x| \rightarrow \infty} V(x)=\infty$,

(ii) $\limsup _{h \rightarrow 0+} 1 / h[V(x+h F(t, x))-V(x)]<0$, for $|x| \geq R, t \in[0, \tau]$,

where $F:[0, \tau] \times \mathbb{R}^{n} \rightarrow \mathbb{R}^{n}$ is a Carathéodory right-hand side in (1.1), and $R>0$ is a constant which may be large. Then system (1.1) is uniformly dissipative.

Proposition 5.2. Let $V_{u}: \mathbb{R}^{n} \rightarrow \mathbb{R}$ be a family of (bounding) functions and $c \in \mathbb{R}$. Set $A=$ $\left[V_{u} \leq c\right]:=\left\{x \in \mathbb{R}^{n} \mid V_{u}(x) \leq c\right\}$; the set $\left[V_{u}>c\right]$ is defined analogously. Assume that $A \subset$ $\mathbb{R}^{n}$ is bounded and that, for each $u \in \partial A$, there exists $\varepsilon>0$ such that $V_{u}$ is locally Lipschitz on $\left[V_{u}>c\right] \cap B(u, \varepsilon)$ and

$$
\limsup _{h \rightarrow 0+} \frac{1}{h}\left[V_{u}(x+h F(t, x))-V_{u}(x)\right] \leq 0, \quad t \in[0, \tau]
$$

for every $x \in\left[V_{u}>c\right] \cap B(u, \varepsilon)$. Then $A$ is positively flow-invariant for (1.1), that is, $x\left(t_{0}\right) \in$ $A$, for every $t_{0} \in[0, \tau]$, implies $x(t) \in A$, for all $t \geq t_{0}$, for solutions $x(\cdot)$ of (1.1).

Hence, we can reformulate Theorem 4.2 in terms of guiding and bounding functions as follows (cf. also Remark 4.3).

Theorem 5.3. Let a locally Lipschitz (guiding) function $V: \mathbb{R}^{n} \rightarrow \mathbb{R}$ exist such that conditions (i), (ii) in Proposition 5.1 are satisfied. Then system (1.1) admits a $\tau$-periodic solution. Moreover, if a compact ENR-set $A \subset \mathbb{R}^{n}$ still exists such that the assumptions of Proposition 5.2 are satisfied with $A=\left[V_{u} \leq c\right]$, for a family of (bounding) functions $V_{u}$ : $\mathbb{R}^{n} \rightarrow \mathbb{R}$, then there exists a $\tau$-periodic solution $x_{0}(\cdot)$ of $(1.1)$, with $x_{0}(t) \in \mathscr{D}$, for all $t \in \mathbb{R}$, and with $x_{0}(0) \in \mathscr{D} \backslash \operatorname{int} A$, where $\mathscr{D}:=\left\{x_{0} \in \mathbb{R}^{n}|| x_{0} \mid<D_{2}\right\}$ (cf. (1.3)), provided $\chi(A) \neq 1$. In the case of uniqueness, the existence of guiding and bounding functions with the above properties implies also at least $1+N\left(\left.\mathrm{id}\right|_{A}\right) \tau$-periodic solutions of $(1.1)$, provided there is no common essential Nielsen class of $\left.T_{\tau}\right|_{\mathbb{R}^{n}}$ and $\left.T_{\tau}\right|_{A}$.

Example 2. Taking in Theorem 5.3 the same $A \subset \mathbb{R}^{n}$ as in Example 1, we obtain obviously again a $\tau$-periodic solution $x_{0}(\cdot)$ of $(1.1)$ with $x_{0}(0) \in \mathscr{D} \backslash \operatorname{int} A$ and, in the case of uniqueness, three $\tau$-periodic solutions of (1.1). 
If the sharp inequality still holds in condition (5.1), then at least three $\tau$-periodic solutions $x_{1}(\cdot), x_{2}(\cdot), x_{3}(\cdot)$ of (1.1) always (i.e., also in the absence of uniqueness) exist such that $x_{1}(t) \in A_{1}, x_{2}(t) \in A_{2}$, and $x_{3}(t) \in \mathscr{D} \backslash A$, for all $t \in \mathbb{R}$.

Remark 5.4. Observe that if a positively flow-invariant compact ENR-set $A \subset \mathbb{R}^{n}$ satisfies $\chi(A) \notin\{0,1\}$ and its boundary $\partial A$ is fixed point free (e.g., if the sharp inequality holds in (5.1)), then at least two $\tau$-periodic solutions of the uniformly dissipative system (1.1) exist (one with values in int $A$ and the second outside of $A$ ). If $A$ is a compact ENR-set and a uniqueness condition holds for (1.1), then we can have at least $1+N\left(\left.\mathrm{id}\right|_{A}\right) \tau$-periodic solutions, provided the assumptions of the last part of Theorem 4.2 or Theorem 5.3 are satisfied.

Remark 5.5. The situation for differential systems in infinite dimensions is still more delicate. Nevertheless, we have at our disposal fixed point theorems like Theorems 2.11 and 2.12 and their multivalued analogies (cf. [2]).

Remark 5.6. All the above conclusions can be extended to the uniformly dissipative systems of inclusions with upper-Carathéodory right-hand sides whose values are convex and compact, because the regularity of the associated Poincare translation operators is the same. They are namely admissible in the sense of the second author. For more details, see [2].

Remark 5.7. It is an open problem whether or not dissipativity of time periodic system (1.1) implies its uniform dissipativity, in the lack of uniqueness. More generally, it is a question, whether or not an analogy of Theorem 4.1 holds with a compact attractor in a weaker sense.

\section{Acknowledgment}

The first author was supported by the Council of Czech Government (MSM 6198959214).

\section{References}

[1] J. Andres, M. Gaudenzi, and F. Zanolin, A transformation theorem for periodic solutions of nondissipative systems, Università e Politecnico di Torino. Seminario Matematico. Rendiconti 48 (1990), no. 2, 171-186 (1992).

[2] J. Andres and L. Górniewicz, Topological Fixed Point Principles for Boundary Value Problems, Topological Fixed Point Theory and Its Applications, vol. 1, Kluwer Academic, Dordrecht, 2003.

[3] J. Andres, L. Górniewicz, and J. Jezierski, Relative versions of the multivalued Lefschetz and Nielsen theorems and their application to admissible semi-flows, Topological Methods in Nonlinear Analysis 16 (2000), no. 1, 73-92.

[4] J. Andres, L. Górniewicz, and M. Lewicka, Partially dissipative periodic processes, Topology in Nonlinear Analysis (Warsaw, 1994) (K. Gęba and L. Górniewicz, eds.), Banach Center Publ., vol. 35, Polish Acad. Sci., Warsaw, 1996, pp. 109-118.

[5] J. Andres and P. Wong, Relative Nielsen theory for noncompact spaces and maps, to appear in Topology and Its Applications.

[6] J. E. Billotti and J. P. LaSalle, Dissipative periodic processes, Bulletin of the American Mathematical Society (New Series) 77 (1971), 1082-1088. 
[7] F. E. Browder, On a generalization of the Schauder fixed point theorem, Duke Mathematical Journal 26 (1959), no. 2, 291-303.

[8] T. A. Burton and S. N. Zhang, Unified boundedness, periodicity, and stability in ordinary and functional-differential equations, Annali di Matematica Pura ed Applicata. Serie Quarta 145 (1986), 129-158.

[9] M. L. Cartwright, Forced oscillations in nonlinear systems, Contributions to the Theory of Nonlinear Oscillations, Annals of Mathematics Studies, no. 20, Princeton University Press, New Jersey, 1950, pp. 149-241.

[10] I. D. Chueshov, Introduction to the Theory of Infinite-Dimensional Dissipative Systems, University Lectures in Contemporary Mathematics, AKTA, Kharkiv, 1999.

[11] G. Fournier, Généralisations du théorème de Lefschetz pour des espaces non-compacts. I. Applications éventuellement compactes, Bulletin de l'Académie Polonaise des Sciences. Série des Sciences Mathématiques, Astronomiques et Physiques 23 (1975), no. 6, 693-699.

[12] _ Généralisations du théorème de Lefschetz pour des espaces non-compacts. II. Applications d'attraction compacte, Bulletin de l'Académie Polonaise des Sciences. Série des Sciences Mathématiques, Astronomiques et Physiques 23 (1975), no. 6, 701-706.

[13] __ Généralisations du théorème de Lefschetz pour des espaces non-compacts. III. Applications asymptotiquement compactes, Bulletin de l'Académie Polonaise des Sciences. Série des Sciences Mathématiques, Astronomiques et Physiques 23 (1975), no. 6, 707-711.

[14] V. M. Geršteǐn, On the theory of dissipative differential equations in a Banach space, Funkcional'nyi Analiz i ego Priloženija 4 (1970), no. 3, 99-100 (Russian).

[15] L. Górniewicz, Topological Fixed Point Theory of Multivalued Mappings, Mathematics and Its Applications, vol. 495, Kluwer Academic, Dordrecht, 1999.

[16] _ On the Lefschetz fixed point theorem, Mathematica Slovaca 52 (2002), no. 2, 221-233.

[17] L. Górniewicz and A. Granas, On a theorem of C. Bowszyc concerning the relative version of the Lefschetz fixed point theorem, Bulletin of the Institute of Mathematics. Academia Sinica 13 (1985), no. 2, 137-142.

[18] A. Granas and J. Dugundji, Fixed Point Theory, Springer Monographs in Mathematics, Springer, New York, 2003.

[19] J. K. Hale, Asymptotic Behavior of Dissipative Systems, Mathematical Surveys and Monographs, vol. 25, American Mathematical Society, Rhode Island, 1988.

[20] J. K. Hale, J. P. LaSalle, and M. Slemrod, Theory of a general class of dissipative processes, Journal of Mathematical Analysis and Applications 39 (1972), no. 1, 177-191.

[21] J. K. Hale and O. Lopes, Fixed point theorems and dissipative processes, Journal of Differential Equations 13 (1973), no. 2, 391-402.

[22] G. S. Jones, Stability and asymptotic fixed-point theory, Proceedings of the National Academy of Sciences of the United States of America 53 (1965), 1262-1264.

[23] N. Levinson, Transformation theory of non-linear differential equations of the second order, Annals of Mathematics. Second Series 45 (1944), no. 4, 723-737.

[24] R. D. Nussbaum, Some asymptotic fixed point theorems, Transactions of the American Mathematical Society 171 (1972), 349-375.

[25] _ The Fixed Point Index and Some Applications, Séminaire de Mathématiques Supérieures, vol. 94, Presses de l'Université de Montréal, Quebec, 1985.

[26] N. Pavel, On dissipative systems, Bollettino della Unione Matematica Italiana. Serie IV 4 (1971), 701-707.

[27] V. A. Pliss, Nonlocal Problems of the Theory of Oscillations, Academic Press, New York, 1966. 


\section{Dissipative systems}

[28] D. Richeson and J. Wiseman, A fixed point theorem for bounded dynamical systems, Illinois Journal of Mathematics 46 (2002), no. 2, 491-495.

[29] U. K. Scholz, The Nielsen fixed point theory for noncompact spaces, Rocky Mountain Journal of Mathematics 4 (1974), 81-87.

[30] T. Yoshizawa, Stability Theory and the Existence of Periodic Solutions and Almost Periodic Solutions, Applied Mathematical Sciences, vol. 14, Springer, New York, 1975.

Jan Andres: Department of Mathematics Analysis, Faculty of Science, Palacký University,

Tomkova 40, 77900 Olomouc-Hejčín, Czech Republic

E-mail address: andres@inf.upol.cz

Lech Górniewicz: Faculty of Mathematics and Informatics, Nicolaus Copernicus University, Chopina 12/18, 87-100 Torun, Poland

E-mail address: gorn@mat.uni.torun.pl 